\title{
Uncharacteristic Presentation of Pituitary Macroadenoma - Our Institutional Experience
}

\section{Sibhi Ganapathy* and Rajesh Nair}

\author{
Department of Neurosurgery, Kasturba Medical College, Manipal, India \\ *Corresponding Author: Sibhi Ganapathy, Department of Neurosurgery, Kasturba Medical College, Manipal, India.
}

Received: July 19, 2019; Published: July 25, 2019

DOI: 10.31080/ASNE.2019.02.0088

\begin{abstract}
Unclear eye signs along with multiple ipsilateral cranial nerve palsies have been usually localized to the cavernous sinus or central skull base chordomas. A rare possibility involves pituitary adenomas, which invade laterally without presenting with the classical triad of visual deficits, hormonal dysfunction or ICP features. The following examples examine the unusual presentations of the disease, along with the importance of imaging modalities, which when examined properly can achieve excellent results for the patients. Keywords: Invasive Pituitary; Sellar Erosion; Lower Cranial Nerve Involvement; Unilateral Ptosis; Pituitary Macroadenoma; Craniopharyngioma
\end{abstract}

\section{Introduction}

Pituitary adenomas usually present to the neurosurgeon with the history of hormonal dysfunction, visual symptoms, classically the bitemporal hemianopia, headache and rarely with sudden onset headache and visual loss in case of apoplexy. Cranial nerve involvement and sellar destruction, though described in literature, are rarely encountered in the average pituitary adenomas seen in the out patient department.

Here we describe our experience in two patients with invasive pituitary macroadenomas. The first case was that of a 46-year old housewife who presented with unilateral ptosis with a dilated pupil and complete ophthalmoplegia. She also had unilateral facial dysaesthesia and lower cranial nerve deficits in the form of palatal palsy and absence of gag with deviation of tongue to the left. Imaging and clinical discussions lead us to a diagnosis of clival chordoma however the final MRI report and pathological diagnosis was that of an invasive pituitary macroadenoma.

The second case presented with progressive painless peripheral loss of vision. She has no features of ICP headache and MRI imaging rendered a diagnosis of craniopharyngioma. The intraoperative findings were suggestive of an invasive pituitary macroadenoma and the final pathological diagnosis confirmed the same.

Lower cranial nerve involvement, sellar destruction and ptosis are rarely associated with pituitary and relatively unheard of associations. Here we describe and discuss this varied presentation of a common clinical entity, interpretation of the imaging peculiarities, treatment outcomes and prognosis.

\section{Case Reports}

Case 1

A 46 year-old housewife presented to us with headache since 4 years and acute exacerbation of headache and sudden onset ptosis since the previous night. She gave a classical history of sudden change in the character of the headache from dull aching to an acute holocranial nature, which had happened overnight, however she had no history of vomiting or blurring of vision or photophobia She gave no history suggestive of ICP headache. She also gave a history of left sided complete ophthalmoplegia with no proptosis or swelling of eyelids or conjunctival cheimosis or redness. There was no history of retrobulbar pain or facial paraesthesia. She gave no history suggestive of visual loss or sudden onset loss of consciousness. There was no historical evidence of any endocrinological abnormality or stalk effect or lower cranial nerve involvement. A clinical diagnosis of a ruptured cavernous segment internal carotid artery (ICA) aneurysm was considered. Other differential diagnoses included cavernous sinus thrombosis, giant posterior communicating artery aneurysm (P.comm) and pituitary apoplexy. Clival chordoma was also considered as a clinical diagnosis, in v/o the lesion being in the clival region with areas of focal destruction.

\section{Neuro-examination}

A neurological examination revealed left sided 3rd, 4th and 6th nerve involvement with complete left sided ophthalmoplegia. Left 5th nerve V1 segment also seemed to be involved with involvement of lower cranial nerves $\left(9^{\text {th }}, 10^{\text {th }}, 11^{\text {th }}\right.$ and $12^{\text {th }}$ nerves on the left side). She had her uvula deviated to the left with no movement of the palate on the left side. Gag was absent on the left side and there 
was deviation of the tongue to the left side. Auscultation showed no bruit over the left eye and motor and sensory system examinations were unremarkable.

Anatomically, the lesion seemed to be a carpeting lesion involving the cavernous sinus on the left side and clivus and the lower cranial nerves within the cisternal spaces since there were no brain stem signs.

\section{Hormonal status}

She was in diabetic ketoacidosis with high urine sugar and proteins. Her hormonal status was deranged, with a prolactin level of $1593 \mathrm{ng} / \mathrm{ml}$ and Serum Cortisol level of $1.610 \mathrm{gm} / \mathrm{dl}$.

Case 2

A 41 year-old housewife presented to us with the history of progressive loss of vision in the left eye since the last 2 months. The visual loss started form the peripheral fields of vision and was painless and gradually progressive. She has consulted an ophthalmologist for the same and had no relief or improvement in her symptoms and since the last 15 days she has had total loss of vision in her left eye. There was perception of light in the left eye, however she was able to count fingers, 4 feet away, in the right eye. She also complained of non-ICP headache, which was more so, on the left side. There was no history of any endocrinological abnormality and she also gave no evidence of any hypothalamic involvement (polyphagia, polydypsia, polyuria, hyperthermia). The clinical differentials considered were: optic chiasmal glioma, pituitary tumor and craniopharyngioma.

\section{Neuro-examination}

Further, on neurological examination, the patient seemed to have normal higher mental function with cranial nerve examination revealing a left side diminution in visual acuity. She had perception of light in the left eye with almost normal visual acuity in the right. Right eye field charting (on confrontation technique) showed a right temporal field defect with no papilledema on fundus examination.

The lesion was thought to be in the supra-sellar region involving the left optic nerve and the chiasm, causing the visual field and acuity defects.

\section{Hormonal status}

Serum cortisol was low $(0.633 \mathrm{gm} / \mathrm{dl})$ with normal TFT (T3 $0.78 \mathrm{ng} / \mathrm{ml}, \mathrm{T} 4-9.17 \mathrm{~g} / \mathrm{dl}$ and TSH $-0.644 \mathrm{IU} / \mathrm{ml}$ ). Rest of her hormonal assessment was within normal limits (FSH $-21.3 \mathrm{mIU} / \mathrm{ml}$, LH $23.5 \mathrm{mIU} / \mathrm{ml}$, Prolactin $7.2 \mathrm{ng} / \mathrm{ml}$ and GH $1.06 \mathrm{ng} / \mathrm{ml}$.
Imaging peculiarities

Case 1:

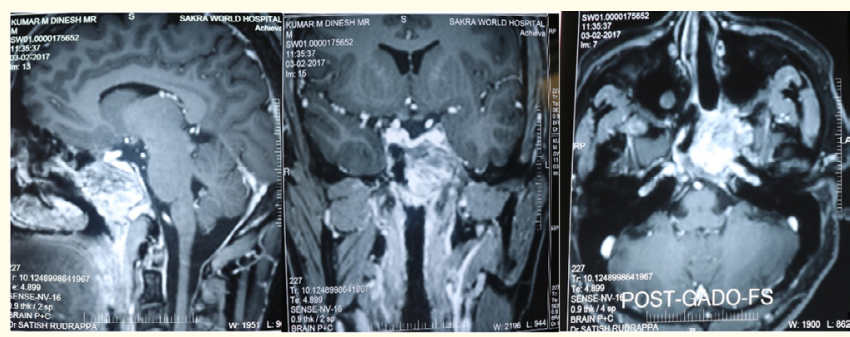

Figure 1a, b and $c$

Magentic resonance imaging (MRI) Brain revealed a large lobulated enhancing lesion with the epicenter within the body of the sphenoid, isointense on T1W and hypointense on T2W and FLAIR, with superior extension into the sella. The lesion seemed to expand the sella and causes focal erosion of the sellar floor, dorsum sellae and posterior clinoid process. The bony destruction was corroborated with computed tomographic (CT) brain bone window cuts. Inferiorly the lesion was obliterating the sphenoid sinus and mildly expanding the body of the sphenoid and clivus with extension into the petrous apex and occipital condyles. Laterally the lesion seemed to be completely encasing the cavernous segment of the ICA and obliterating the left orbital apex with extension into the superior and inferior orbital fissure. Pituitary gland could not be separately delineated from the lesion. Possibilities considered include (1) Pituitary macroadenoma (2) Malignant etiology (lymphoma/plasmacytoma)

\section{Case 2:}
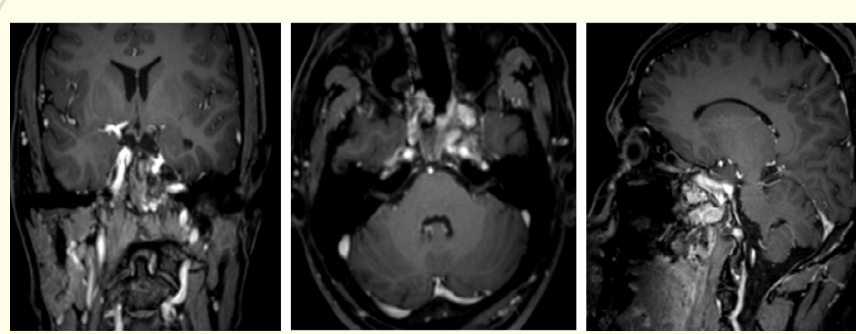

Figure 2a, $b$ and $c$

CT Brain (Plain) showed an extra axial lesion in the suprasellar region with a diffuse intrasellar component with multiple cystic and solid areas within the lesion with no evidence of calcification. Superiorly the lesion was involving the optic chiasm but not extending in to the third ventricle and causing obstructive hydro- 
cephalus. The lesion had also eroded the bony sella turcica. Possibilities considered were (1) Craniopharyngioma, (2) Pituitary macroadenoma.

Outside done MRI Brain showed a hypo to isointense lesion on T1W with heterogenous intensity on T2W with enhancement on contrast and presence of solid and cystic component. Bilateral ICAs seemed to be encased within the lesion and a radiological diagnosis of craniopharyngioma was considered.

Operative procedure

Both the patients in the series underwent a standard transnasal trans sphenoidal approach and gross total excision of the tumor. The final histopathology was reported as invasive pituitary macroadenoma. Post excision, abdominal fat pad graft and fibrin glue was placed with gelfoam layers to prevent postoperative CSF leak. Both patients had no post op CSF rhinorrhea and had an unremarkable postoperative period and were discharged with hormonal levels returning to normal levels.

\section{Discussion}

Pituitary tumors rarely present with cranial nerve deficits [1-5] and are usually considered as differentials when a patient presents with painless progressive peripheral visual loss. In our cases, the first scenario was that of sudden onset ptosis, in the absence of apoplexy $[4,5]$ and in the second case there seemed to be calcification, with cystic component in a sellar-suprasellar lesion with progressive painless visual loss, which was suggestive of an adult variant of craniopharyngioma. These cranial nerve deficits are usually reversible with early diagnosis and intervention [4]. The presence of calcification and erosion of the sellar with extensive involvement of the lower cranial nerves have never been associated with even invasive pituitary tumors. Isolated third nerve involvement, in pituitary adenomas with lateral extension, have been described in literature [6,7]. The radiological picture of a solid and cystic tumor occupying the sellar and supra sellar region also makes one consider craniopharyngiomas over an invasive pituitary tumor.

\section{Conclusion}

The appropriately wry adage, 'tumors don't read textbooks' has been validated once again, with unusual and complicated presentations of the above disease. Careful preoperative imaging and meticulous technique often yields good results.

\section{Bibliography}

1. Saul RF and Hilliker JK. "Third nerve palsy: the presenting sign of a pituitary adenoma in five patients and the only neurological sign in four patients". Journal of Neuro-Ophthalmology 5 (1985): 185-193.

2. Wykes WN. "Prolactinoma presenting with intermittent third nerve palsy". British Journal of Ophthalmology 70 (1986): 706-707.

3. Yen MY., et al. "Ptosis as the early manifestation of pituitary tumour". British Journal of Ophthalmology 74.3 (1990): 188191.

4. Rossitch E Jr., et al. "Isolated oculomotor nerve palsy following apoplexy of a pituitary adenoma". Journal of Neurosurgical Sciences 36.2 (1992): 103-105.

5. Lau KK., et al. "Isolated bilateral oculomotor nerve palsy in pituitary apoplexy: case report and review". British Journal of Neurosurgery 21.4 (2007): 399-402.

6. Lenthall RK and Jaspan T. "A case of isolated third nerve palsy with pupil involvement". The British Journal of Radiology 73 (2000): 569-570.

7. Cano M., et al. "Pituitary adenoma presenting as painful intermittent third nerve palsy". Headache 29 (1989): 451-452.

\section{Volume 2 Issue 8 August 2019 (C) All rights are reserved by Sibhi Ganapathy and Rajesh Nair.}

